\title{
Marie Madeleine en Occident : les dynamiques de la sainteté dans la Bourgogne des $\mathrm{IX}^{\mathrm{e}}-\mathrm{XV}^{\mathrm{e}}$ siècles
}

Thèse de doctorat de l'université d'Avignon et des Pays de Vaucluse (CIHAM, UMR 5648), sous la direction de Guy Lobrichon et de Daniel Russo. Soutenue le 11 décembre 2012

\section{Raphaëlle Taccone}

\section{(2) OpenEdition}

\section{Journals}

\section{Édition électronique}

URL : https://journals.openedition.org/cem/13033

DOI : 10.4000/cem.13033

ISSN : 1954-3093

Éditeur

Centre d'études médiévales Saint-Germain d'Auxerre

\section{Référence électronique}

Raphaëlle Taccone, «Marie Madeleine en Occident : les dynamiques de la sainteté dans la Bourgogne des $\mathrm{x}^{\mathrm{e}}-\mathrm{xv} \mathrm{e}^{\mathrm{e}}$ siècles », Bulletin du centre d'études médiévales d'Auxerre | BUCEMA [En ligne], 17.1 | 2013, mis en ligne le 03 juin 2013, consulté le 22 septembre 2022. URL : http://journals.openedition.org/cem/ 13033 ; DOI : https://doi.org/10.4000/cem.13033

Ce document a été généré automatiquement le 22 septembre 2022.

\section{cc) (†)}

Creative Commons - Attribution - Pas d'Utilisation Commerciale - Partage dans les Mêmes Conditions 4.0 International - CC BY-NC-SA 4.0

https://creativecommons.org/licenses/by-nc-sa/4.0/ 


\section{Marie Madeleine en Occident : les dynamiques de la sainteté dans la Bourgogne des $\mathrm{IX}^{\mathrm{e}}-\mathrm{XV}^{\mathrm{e}}$ siècles}

Thèse de doctorat de l'université d'Avignon et des Pays de Vaucluse (CIHAM, UMR 5648), sous la direction de Guy Lobrichon et de Daniel Russo. Soutenue le 11 décembre 2012

\section{Raphaëlle Taccone}

1 La thèse intitulée Marie Madeleine en Occident: les dynamiques de la sainteté dans la Bourgogne des $I X^{e}-X V^{e}$ siècles a été soutenue le 11 décembre 2012 à l'université d'Avignon et des Pays de Vaucluse. Elle porte sur l'étude du culte de Marie Madeleine au sein de l'ancien duché de Bourgogne et de ses comtés périphériques. La période chronologique retenue se situe ainsi des prémisses d'une dévotion envers la Madeleine (IX ${ }^{\mathrm{e}}$ siècle) au plein épanouissement de son culte $\left(\mathrm{xV}^{\mathrm{e}}\right.$ siècle). La thèse tente de définir pourquoi et comment cette sainte est devenue l'enjeu d'un culte illustre en

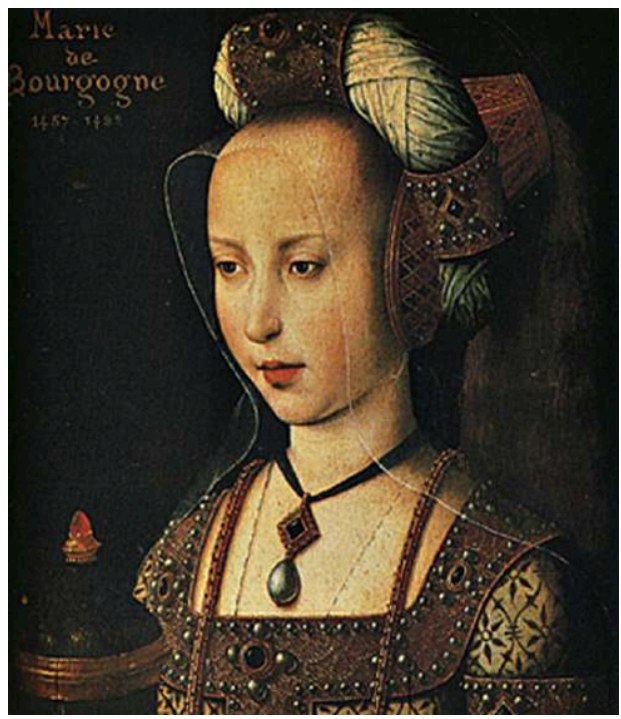
Bourgogne. Ce culte reste-t-il l'apanage du monastère de Vézelay tout au long du Moyen Âge ou est-il absorbé par d'autres pouvoirs?

Dans le prolongement d'une réflexion amorcée lors d'un master études médiévales, la thèse tente de combler un vide sur le sujet, en envisageant le rôle déterminant du pouvoir ducal de Bourgogne dans la promotion du culte magdalénien. Bien après les travaux pionniers de Victor Saxer ${ }^{1}$, aujourd'hui dépassés, cette thèse remet en cause 
l'idée d'un déclin du culte bourguignon de la Madeleine dès le troisième quart du $\mathrm{XIII}^{\mathrm{e}}$ siècle et conforte l'idée d'un rayonnement régional jusqu'à la fin du $\mathrm{Xv}^{\mathrm{e}}$ siècle. Elle aborde le culte de sainte Marie Madeleine en tant qu'enjeu d'une politique monastique puis princière, pleinement capté par le pouvoir ducal de Bourgogne dès le troisième quart du XIV ${ }^{\mathrm{e}}$ siècle. Résolument pluridisciplinaire, la thèse explore ainsi une grande diversité de sources historiques - hagiographiques, liturgiques, hagiotoponymiques, iconographiques... - et s'inscrit dans le champ de l'histoire religieuse de la Bourgogne.

3 L'étude s'articule autour de quatre parties majeures. La première partie aborde les sources du culte de la sainte, en distinguant celles qui traitent de l'histoire du personnage de la Madeleine et celles relatives à son culte. L'étude observe les évangiles canoniques, la littérature gnostique, la patristique et le dossier hagiographique élaboré au monastère de Vézelay. Elle présente également le théâtre des Passions, plus particulièrement la Passion de Semur-en-Auxois ${ }^{2}$, composée à la fin du XIV siècle, où la sainte apparaît comme la femme forte des Évangiles. La première partie propose ainsi de définir les contours de l'identité de Marie Madeleine et aborde la construction de son culte.

4 La deuxième partie de la thèse, fondée sur une perspective liturgique, observe la mise en place du culte de la Madeleine en Bourgogne. Un premier chapitre étudie la place de la sainte dans l'année liturgique. L'étude démontre, que, dès le $\mathrm{vI}^{\mathrm{e}}$ siècle et jusqu'à la fin du Moyen Âge, Marie Madeleine est honorée par des fêtes majeures et mineures ${ }^{3}$. La fête du 22 juillet, qui supplante les autres dès la première moitié du XI ${ }^{e}$ siècle, est très largement relayée en Bourgogne. À l'abbaye de Vézelay, la fête est précédée par une vigile et augmentée par une octave ${ }^{4}$. Au sein du bréviaire d'été du duc Philippe le Bon ${ }^{5}$, l'office du 22 juillet De sancta Maria Magdalena condense des pièces liturgiques spécialement lues en ce jour et d'autres empruntées à l'office pascal, comme Victime paschali laudes. Enfin, une attention particulière a été portée aux prières spécialement dévolues à la sainte. Si elles sont le plus souvent insérées dans des livres d'heures composés pour de nobles dames, l'étude a démontré la présence tout à fait singulière d'une prière De Marie Magdelaine au sein d'un livre de prières du duc de Bourgogne Philippe le Bon ${ }^{6}$. Cette dernière présente la particularité de se terminer par un court Ave Marie Magdelenae. Le second chapitre de cette partie présente le discours exégétique relatif au personnage de la Madeleine. Il résume les écrits patristiques sur le sujet et envisage ensuite l'homilétique bourguignonne. L'étude insiste sur la Madeleine nouvelle des Évangiles touchée par la grâce du rachat, véritable via media entre Ève et la Vierge Marie. Sainte Marie Madeleine incarne pleinement une sainte médiatrice, pouvant intercéder entre Dieu et les fidèles ${ }^{7}$. Du XI ${ }^{\mathrm{e}}$ siècle à la fin du Moyen Âge, la Madeleine est unanimement célébrée comme une figure du repentir qui lave la culpabilité de ses fautes dans le fleuve de ses larmes. Enfin, l'étude aborde Marie Madeleine comme un modèle de comportement proposé par l'Église et comme une figure inspiratrice pour les femmes. Abordant la sainteté féminine, la thèse insiste sur le besoin d'imiter par le sacrifice des larmes et les jeûnes la Madeleine mystique. Comme l'a démontré Susan Haskins ${ }^{8}$, dès le xiII ${ }^{\mathrm{e}}$ siècle, le modèle de la Madeleine prédicatrice incite surtout une vague de femmes visionnaires à pratiquer la parole publique grâce au don charismatique de la prophétie.

5 La troisième partie de la thèse, la plus conséquente, ouvre sur les différentes formes et pratiques du culte magdalénien. Tout d'abord initié par l'abbé Geoffroi de Vézelay, dès le second quart du XI ${ }^{\mathrm{e}}$ siècle, le culte est récupéré par le pouvoir ducal de Bourgogne, à 
compter du troisième quart du XIII siècle. De 1364 à la fin $d u x^{e}$ siècle, il est pleinement revendiqué par la lignée des Valois, de Philippe le Hardi à Marie de Bourgogne.

Pour mesurer la dévotion en termes généraux, un premier chapitre observe la popularité du culte de la Madeleine, du cercle privé au cadre collectif (confréries, corporations de métiers). Dans un second temps, ce même chapitre expose l'amplification de la fête du 22 juillet par la tenue de foires ou encore par le jeu du théâtre des Passions. Les deux chapitres suivants présentent respectivement le culte

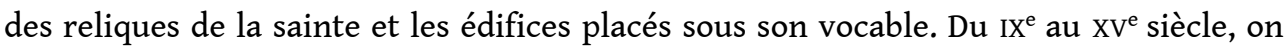
relève une dizaine de reliques et plus de quatre-vingt-dix sanctuaires magdaléniens. Ces mentions se concentrent principalement au sein et dans la périphérie des villes de Sens, Auxerre, Nevers et Dijon, le long de la vallée de la Loire et de la Saône. Ces foyers dévotionnels, circonscrivant Vézelay, assurent la pérennité du culte magdalénien. Si le culte de Marie Madeleine est particulièrement actif au sein du duché de Bourgogne et de ses comtés périphériques, il peine à acquérir un rayonnement au sein des Pays-Bas bourguignons. Toutefois, il convient de souligner la fondation de l'hôpital Sainte-Marie Madeleine à Ath par le duc Philippe le Bon après l'incendie de la ville en $1435^{9} \mathrm{et}$, le 27 avril 1485, l'élévation du couvent des repenties de la Madeleine à Mons par la duchesse Marguerite de York ${ }^{10}$. Enfin, le quatrième chapitre aborde les représentations bourguignonnes de sainte Marie Madeleine. Le corpus iconographique, exposant distinctement l'art monumental et les manuscrits enluminés, a démontré que ces images intégraient une thématique pénitentielle $d u x^{e}$ à la fin $d u x{ }^{e}{ }^{e}$ siècle, puis une thématique axée sur la mort du Christ du XIV à la fin du Xve siècle. L'étude a également souligné la petitesse de la sainte et parfois l'absence de son nimbe au sein de certaines images, écho d'une Madeleine humanisée, offerte comme modèle de contemplation aux pêcheurs.

7 La quatrième partie de la thèse aborde les enjeux politiques du culte de la sainte. Le premier chapitre présente le culte magdalénien comme enjeu d'une stratégie monastique de l'abbaye de Vézelay - déjà bien exploré par les travaux de René Louis ${ }^{11}$ -, puis d'une politique princière pour le pouvoir ducal de Bourgogne. Dans un second temps, la thèse envisage le renouveau du culte de la Madeleine à la cour de Bourgogne, à partir du règne de Philippe le Hardi (1364-1404). Elle identifie la dévotion constante des ducs Philippe le Bon (1419-1467) et Charles le Téméraire (1467-1477). Enfin, ce chapitre traite de la piété des duchesses de Bourgogne envers la noble dame de Magdala et insiste sur la dévotion active des femmes de la lignée de Valois, de Marguerite de York (1468-1477) à Marie de Bourgogne (1477-1482). La thèse observe notamment que la mode de se faire représenter sous les traits de Marie Madeleine est initiée par la duchesse Marguerite de York, puis se répercute dans l'art du portrait auprès d'une aristocratie féminine jusqu'au début du Xvi ${ }^{\mathrm{e}}$ siècle (fig. 1). 
Fig. 1 - Portrait de Marie de Bourgogne, vers 1480. Chantilly, musée Condé (cl. R. Taccone). La duchesse Marie de Bourgogne (1477-1482) est figurée sous les traits de la Madeleine portant son attribut traditionnel, un vase à onguent.

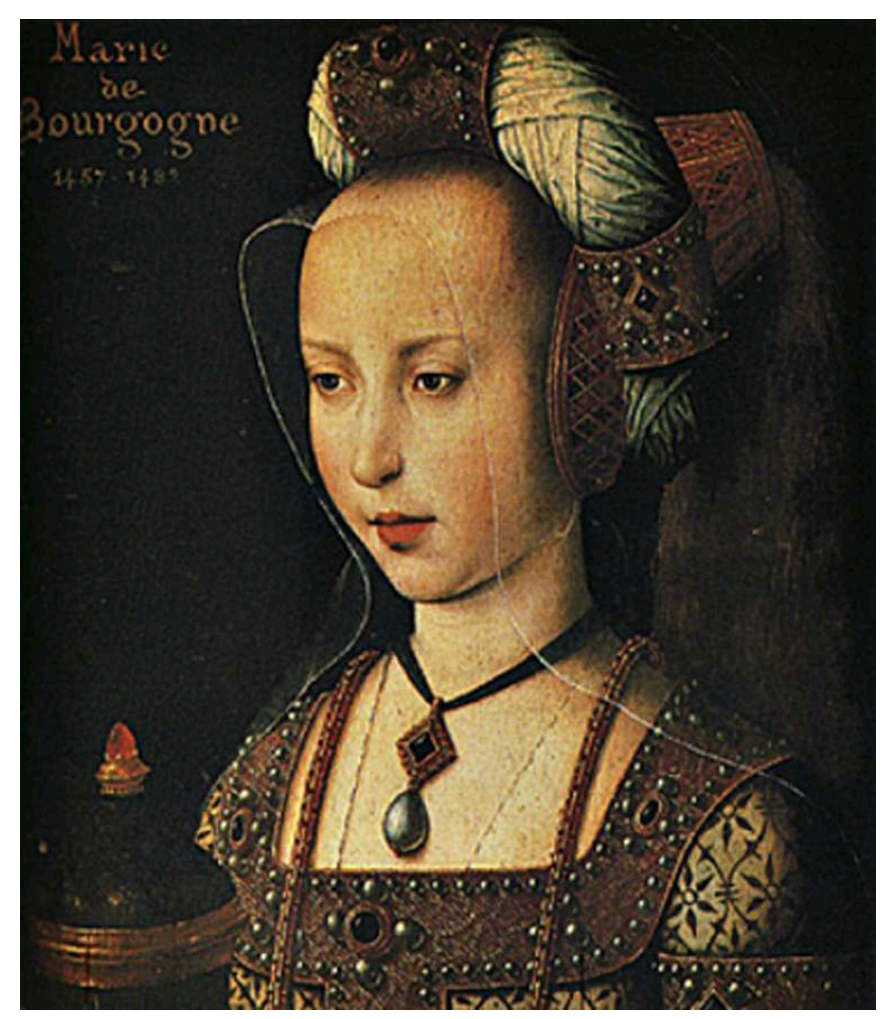

8 Le chapitre suivant, " un culte politisé », révèle une compétition croissante entre le pouvoir ducal de Bourgogne et le pouvoir comtal de Provence et observe l'instrumentalisation du culte magdalénien à des fins de légitimation de leur autorité respective. Si les ducs de Bourgogne honorent Girart de Roussillon - le fondateur du monastère de Vézelay et le pieux commanditaire du vol des reliques de la Madeleine comme l'illustre ancêtre de la famille ducale ${ }^{12}$, il convient d'observer que les comtes de Provence louent très largement Charles de Salerne, qui découvrit le tombeau supposé de la Madeleine à Saint-Maximin. Du troisième quart du XIII ${ }^{\mathrm{e}}$ siècle à la fin $\mathrm{du} \mathrm{XV} \mathrm{Xv}^{\mathrm{e}}$ siècle, l'étude démontre les rivalités croissantes entretenues entre ces deux lignages, particulièrement accrues sous les règnes du duc de Bourgogne Philippe le Bon et du comte de Provence René le Bon. Enfin, le dernier chapitre de la thèse révèle comment le culte de la sainte fut promu en Bourgogne. Il observe la permanence de réseaux cultuels autour de l'abbaye de Vézelay et dans l'aire d'action des ducs bourguignons. Concernant ce dernier point, l'étude atteste que le culte de Marie Madeleine fut largement favorisé par une piété filiale et familiale au sein de la lignée des Valois et que, selon le principe de l'amicitia, le culte se répercuta au sein de la cour ducale.

Dans son ensemble, la thèse entend démontrer la vitalité du culte de Marie Madeleine au sein du duché de Bourgogne et de ses comtés périphériques. Elle propose également d'identifier les fonctions de la sainteté magdalénienne - sainte tutélaire, patronne, médiatrice, thaumaturge ou encore dynastique. D'une politique monastique aux stratégies princières, le culte de la sainte se révèle comme le pieux héritage du comte Girart de Roussillon, porteur d'une identité religieuse régionale destinée à être promue. Reçu : 24 janvier 2013 - Accepté : 13 mai 2013 


\section{NOTES}

1. V. SAXER, Le culte de sainte Marie Madeleine en Occident. Des origines à la fin du Moyen Âge, Paris, 1959, 2 vol.

2. PARIS, BnF, ms. fr. 904 ; The Passion de Semur, éd. L. MUIR, Leeds, 1981.

3. Fêtes majeures : à Pâques (dès le $\mathrm{VI}^{\mathrm{e}}$ siècle), le 19 janvier ( $\mathrm{du} \mathrm{VI}^{\mathrm{e}}$ siècle jusqu'à la première moitié $\mathrm{du} \mathrm{XII}$ esiècle), le 22 juillet (à partir du viII ${ }^{\mathrm{e}}$ siècle). Fêtes mineures : le 19 mars (dès la

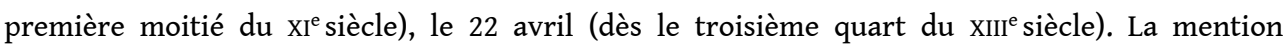
calendaire du 19 janvier doit être observée avec prudence, car elle résulte d'une erreur d'un moine copiste, V. SAXER, Le culte..., op. cit., t. 1, p. 36-37.

4. LYON, BM, ms. 555 , fol. $317 \mathrm{v}^{\circ}-322 \mathrm{r}^{\circ}$.

5. BRUXELLES, KBR, ms. 9026 , fol. $321 \mathrm{v}^{\circ}-325 \mathrm{r}^{\circ}$.

6. PARIS, BnF, ms. naf 16428 , fol. $20 \mathrm{v}^{\circ}-21 \mathrm{r}^{\circ}$.

7. X. BARRAL I ALTET, « Georges Duby et l'art médiéval », in C. DUHAMEL-AMADO et G. LOBRICHON (dir.), Georges Duby. L'écriture de l'histoire, Bruxelles, 1996, p. 73-76.

8. S. HASKINS, Mary Magdalen. Myth and Metaphor, New York, 1993 ; voir également, B. KIENZLE et P. WALKER (Éd.), Women Preachers and Prophets through two Millennia of Christianity, Berkeley, 1998.

9. J.-M. CAuchiES, La législation princière pour le comté de Hainaut. Ducs de Bourgogne et premiers Habsbourg (1427-1506), Bruxelles, 1982, p. 168.

10. E. BOUSMAR, «Marguerite de York et les putains de Mons, entre charité dévote et offensive moralisatrice (1481-1485). Autour d'une fondation de repenties», in J.-M. CAUCHIEs (dir.), Marguerite de York et son temps, Neuchâtel, 2004, p. 75-96.

11. R. LouIs, Girart, comte de Vienne (819-877) et ses fondations monastiques, Auxerre, 1946 ; ID., Girart, comte de Vienne, dans les chansons de geste. Girart de Vienne, Girart de Fraite, Girart de Roussillon, Auxerre, 1947, 2 vol.

12. Y. LACAZE, "Le rôle des traditions dans la genèse d'un sentiment national au $\mathrm{XV}^{\mathrm{e}}$ siècle ", Bibliothèque de l'École des chartes, 1971, p. 308-385.

\section{INDEX}

Mots-clés : culte, duc de Bourgogne, Marie-Madeleine (sainte), hagiographie Index géographique : France/Saint-Maximin, France/Vézelay, France/Bourgogne 